\title{
A four-year surveillance program for detection of Plasmodium falciparum chloroquine resistance in Honduras
}

\author{
Gustavo A Fontecha ${ }^{1} /{ }^{+}$, Ana L Sanchez ${ }^{2}$, Meisy Mendoza ${ }^{3}$, Engels Banegas ${ }^{4}$, Rosa E Mejía-Torres ${ }^{5}$ \\ ${ }^{1}$ Microbiology Research Institute, National Autonomous University of Honduras, Tegucigalpa, Honduras \\ ${ }^{2}$ Department of Health Sciences, Faculty of Applied Health Sciences, Brock University, Saint Catharines, ON, Canada \\ ${ }^{3}$ National Malaria Laboratory, National Department of Surveillance ${ }^{4}$ National Malaria Program, Department of Health Promotion, \\ Ministry of Health-Honduras, Tegucigalpa, Honduras ${ }^{5}$ Panamerican Health Organization-Honduras, Tegucigalpa, Honduras
}

Countries could use the monitoring of drug resistance in malaria parasites as an effective early warning system to develop the timely response mechanisms that are required to avert the further spread of malaria. Drug resistance surveillance is essential in areas where no drug resistance has been reported, especially if neighbouring countries have previously reported resistance. Here, we present the results of a four-year surveillance program based on the sequencing of the pfcrt gene of Plasmodium falciparum populations from endemic areas of Honduras. All isolates were susceptible to chloroquine, as revealed by the pfcrt "CVMNK” genotype in codons 72-76.

Key words: Plasmodium falciparum - chloroquine - resistance

Honduras, which is located in Central America, has been endemic for malaria for many recorded centuries. Fortunately, because of national control efforts, the country has recently attained the goal of reducing the incidence of malaria by at least $75 \%$ by 2015 (NML 2011, WHO 2012). However, more than 5,000 cases of malaria are still being reported annually; the majority of these cases are caused by Plasmodium vivax and $12-15 \%$ are due to Plasmodium falciparum (NML 2011).

In Central America, falciparum malaria is treated with chloroquine (CQ) because no instances of drug resistance have yet been reported in the region (Jovel et al. 2011, Mejía-Torres et al. 2013). A potential threat to the current malaria control success in Honduras is that CQresistant $P$. falciparum strains have already been identified in neighbouring regions over the past several years (Echeverry et al. 2013); more recently, a resistant phenotype (CVIET) was detected in two of the 53 isolates obtained from the border region of Nicaragua (Sridaran et al. 2014). It is widely recognised that global commerce and international travel provide opportunities for the spread of infectious pathogens across international borders.

CQ resistance is conferred by mutations in the CQ transporter gene $(p f c r t)$. A five-amino acid sequence located in positions 72-76 of this gene is the main determinant for CQ susceptibility vs. resistance. In addition to the CQ susceptible wild type strain (CVMNK), at least four mutant lineages have been associated with a resistant phenotype, namely CVIET, CVIDT, SVMNT and

doi: $10.1590 / 0074-0276140067$

Financial support: DICU-UNAH, PAHO-Honduras, NML

+ Corresponding author: gustavo.fontecha@unah.edu.hn

Received 23 February 2014

Accepted 22 May 2014
CVMNT (Takahashi et al. 2012). Monitoring genotype changes in circulating parasites could be useful as an effective early warning system that can help develop the timely response mechanisms required to avert the further spread of the resistant strains. This might prove instrumental in preventing the widespread distribution of resistant strains, as observed decades ago on other continents (Wootton et al. 2002, Mita et al. 2009, OgouyemiHounto et al. 2013, Thomsen et al. 2013).

For this reason, a surveillance program has been implemented in Honduras since 2010 to monitor for drug resistance in circulating P. falciparum isolates (NML 2011).

A total of 160 samples, from the same number of patients, collected between 2010-2013 were analysed (68 in 2010, 28 in 2011 and 32 each in 2012 and 2013). The data from the 2010 samples have been published elsewhere (Mejía-Torres et al. 2013). Blood samples were collected from febrile patients seeking medical attention at primary health centres across the country. Upon malaria diagnosis, patients were asked for consent to be included in the drug resistance surveillance program. Consenting patients provided a blood sample through a finger prick and the blood was blotted onto a paper filter. The 160 samples represented a randomised $10 \%$ of samples diagnosed as $P$. falciparum and received for quality control at the National Malaria Laboratory (NML) in Tegucigalpa, the capital city. These samples belonged to patients residing in six endemic departments in the country. Laboratory work was conducted at the Teasdale-Corti Laboratory (School of Microbiology) and the NML.

DNA was extracted using Chelex-100 (Bio-Rad Laboratories, Inc, USA) (de Lamballerie et al. 1992). The microscopic diagnosis of the parasite species was confirmed through amplification of the 18Sr gene (Singh et al. 1999). The $P$. falciparum pfcrt gene was amplified using nested polymerase chain reaction (PCR) as previously described (Mejía-Torres et al. 2013), based on the technique developed by Griffing et al. (2010). Amplicons were observed on $1 \%$ et-br agarose gels. PCR products 
were purified according to the manufacturer's instructions (Promega) and sequenced at the Macrogen facilities (macrogenusa.com) using the primer 5'-TTTTTCCCTTGTCGACCTTAAC-3'. Chromas Pro and Mega5 software were used for sequence analysis. Positions 7276 of pfcrt exon two were evaluated.

All of the samples $(n=160)$ collected during the four consecutive years of surveillance revealed the $p f c r t$ "CVMNK" genotype in codons 72-76 that is associated with CQ susceptibility. This genetic approach supports the hypothesis that $P$. falciparum CQ resistant strains have neither emerged locally nor been introduced into the country. These findings indicate that CQ is still efficacious for the treatment of falciparum malaria in Honduras, which is congruent with the clinical experience described by local health care professionals and with the reports from the national health authorities.

Our findings are excellent news for the country and the Central American region, but they do not deny the potential for resistant strains emerging and being imported into the Honduran territory, as demonstrated by the detection of two isolates with the resistance allele CVIET in Nicaragua (Sridaran et al. 2014). Those isolates were recovered from the Nicaraguan North Atlantic Autonomous Region, which shares a relatively homogeneous parasite population with its neighbouring Honduran Moskitia region (Larranaga et al. 2013). In addition, to our knowledge, Honduran health officials have detected three cases of patients infected in Africa with resistant strains of $P$. falciparum (unpublished data); one of these patients died before he could receive proper treatment.

In conclusion, the monitoring of the circulating phenotypes and genotypes in the country is necessary to ensure Honduras's progress towards the elimination of malaria that could be halted by the development of drug-resistant parasites.

\section{REFERENCES}

de Lamballerie X, Zandotti C, Vignoli C, Bollet C, de Micco P 1992. A one-step microbial DNA extraction method using "Chelex 100" suitable for gene amplification. Res Microbiol 143: 785-790.

Echeverry DF, Nair S, Osorio L, Menon S, Murillo C, Anderson TJ 2013. Long term persistence of clonal malaria parasite Plasmodium falciparum lineages in the Colombian Pacific region. BMC Genet 14: 2.

Griffing S, Syphard L, Sridaran S, McCollum AM, Mixson-Hayden T, Vinayak S, Villegas L, Barnwell JW, Escalante AA, Udhayakumar V 2010. pfmdr1 amplification and fixation of pfcrt chloroquine resistance alleles in Plasmodium falciparum in Venezuela. Antimicrob Agents Chemother 54: 1572-1579.
Jovel IT, Mejía RE, Banegas E, Piedade R, Alger J, Fontecha G, Ferreira PE, Veiga MI, Enamorado IG, Bjorkman A, Ursing J 2011. Drug resistance associated genetic polymorphisms in Plasmodium falciparum and Plasmodium vivax collected in Honduras, Central America. Malar J 10: 376.

Larranaga N, Mejía RE, Hormaza JI, Montoya A, Soto A, Fontecha GA 2013. Genetic structure of Plasmodium falciparum populations across the Honduras-Nicaragua border. Malar J 12: 354.

Mejía-Torres RE, Banegas EI, Mendoza M, Diaz C, Bucheli ST, Fontecha GA, Alam MT, Goldman I, Udhayakumar V, Zambrano JO 2013. Efficacy of chloroquine for the treatment of uncomplicated Plasmodium falciparum malaria in Honduras. Am J Trop Med Hyg 88: 850-854.

Mita T, Tanabe K, Kita K 2009. Spread and evolution of Plasmodium falciparum drug resistance. Parasitol Int 58: 201-209.

NML - National Malaria Laboratory 2011. National protocol for routine surveillance of Plasmodium falciparum antimalaric drug resistance, National Surveillance Laboratory/Honduras Ministry of Health, Tegucigalpa, $16 \mathrm{pp}$.

Ogouyemi-Hounto A, Ndam NT, Gazard DK, d'Almeida S, Koussihoude L, Ollo E, Azagnandji C, Bello M, Chippaux JP, Massougbodji A 2013. Prevalence of the molecular marker of Plasmodium falciparum resistance to chloroquine and sulphadoxine/ pyrimethamine in Benin seven years after the change of malaria treatment policy. Malar J 12: 147.

Singh B, Bobogare A, Cox-Singh J, Snounou G, Abdullah MS, Rahman HA 1999. A genus and species-specific nested polymerase chain reaction malaria detection assay for epidemiologic studies. Am J Trop Med Hyg 60: 687-692.

Sridaran S, Rodriguez B, Soto AM, de Oliveira AM, Udhayakumar V 2014. Molecular analysis of chloroquine and sulfadoxine-pyrimethamine resistance-associated alleles in Plasmodium falciparum isolates from Nicaragua. Am J Trop Med Hyg 90: 840-845.

Takahashi N, Tanabe K, Tsukahara T, Dzodzomenyo M, Dysoley L, Khamlome B, Sattabongkot J, Nakamura M, Sakurai M, Kobayashi J, Kaneko A, Endo H, Hombhanje F, Tsuboi T, Mita T 2012. Large-scale survey for novel genotypes of Plasmodium falciparum chloroquine-resistance gene pfcrt. Malar J 11: 92.

Thomsen TT, Madsen LB, Hansson HH, Tomas EV, Charlwood D, Bygbjerg IC, Alifrangis M 2013. Rapid selection of Plasmodium falciparum chloroquine resistance transporter gene and multidrug resistance gene-1 haplotypes associated with past chloroquine and present artemether-lumefantrine use in Inhambane district, southern Mozambique. Am J Trop Med Hyg 88: 536-541.

WHO - World Health Organization 2012. World Malaria Report 2012. [cited 2014 May 22]. Available from: who.int/malaria/publications/world_malaria_report_2012/en/.

Wootton JC, Feng X, Ferdig MT, Cooper RA, Mu J, Baruch DI, Magill AJ, Su XZ 2002. Genetic diversity and chloroquine selective sweeps in Plasmodium falciparum. Nature 418: 320-323. 